\title{
Germanica
}

\section{«Kässpatzen, Dampfnudeln und Gamsfiletsulz».}

Der traditionalistische Ernährungsdiskurs im Provinzkrimi und seine Funktion für die Konstruktion regionaler Identität

"Kässpatzen, Dampfnudeln et Gamsfiletsulz ». Le discours traditionnel sur

l'alimentation dans le roman policier de province et sa fonction pour la construction de l'identité régionale

"Kässpatzen, Dampfnudeln and Gamsfiletsulz". Traditional food discourse in the provincial crime novel and its function in the construction of regional identity

Julie Bartosch

\section{(2) OpenEdition}

Journals

Édition électronique

URL : http://journals.openedition.org/germanica/3070

DOI : 10.4000/germanica.3070

ISSN : 2107-0784

Éditeur

Université de Lille

Édition imprimée

Date de publication : 30 décembre 2015

Pagination : 159-174

ISBN : 9782913857360

ISSN : 0984-2632

Référence électronique

Julie Bartosch, « «Kässpatzen, Dampfnudeln und Gamsfiletsulz». », Germanica [Online], 57 | 2015,

Online erschienen am: 30 Dezember 2017, abgerufen am 06 Oktober 2020. URL : http://

journals.openedition.org/germanica/3070; DOI : https://doi.org/10.4000/germanica.3070

(C) Tous droits réservés 


\title{
«Kässpatzen, Dampfnudeln und Gamsfiletsulz». \\ Der traditionalistische Ernährungsdiskurs im Provinzkrimi und seine Funktion für die Konstruktion regionaler Identität
}

\author{
Julie BARTOSCH \\ Universität Wien
}

\section{Das Erzählen des Essens - mehr als Dekor}

Im deutschsprachigen Kriminalroman ist eine Etablierung des Motivs von Essen und Trinken als manifester Erzählstrang erst in den späten siebziger und frühen achtziger Jahren innerhalb des Subgenres Wiener Krimi anzusetzen. Seither lässt sich sein stetiger Bedeutungszuwachs beobachten, der im Provinzkrimi, dem gegenwärtig produktivsten und erfolgreichsten Subgenre, das bisherige Maximum erreicht. Wie Kriminalliteratur insgesamt in der literaturwissenschaftlichen Forschung ein Schattendasein fristet, ist auch dieser Aspekt bislang kaum untersucht. Als Studien, die konkret dem Motiv des Essens und Trinkens im Krimi gelten, sind lediglich zwei Aufsätze von Daniela Strigl und Julika Griem nennenswert ${ }^{1}$. Ansonsten sind

1. - Julika Griem: „Mordshunger. Kulinarische Konjunkturen in Kriminalliteratur“, in: Zeitschrift für Kulturwissenschaften, 2012, 1, 83-95; Daniela Strigl: „Der Hedonismus

und der Tod. Warum in Krimis so viel gegessen und getrunken wird“, in: Friedrich Aspetsberger, Daniela Strigl (Hg.): Ich kannte den Mörder, wußte nur nicht wer er war. 
Auseinandersetzungen mit dieser Thematik lediglich als Teilaspekte von allgemeiner gehaltenen Untersuchungen, etwa zu einer Krimiserie in ihrer Gesamtheit, anzutreffen².

In den von Griem und Strigl offerierten Erklärungsmodellen, die als großräumiger Befund an Texten verschiedenster Sprachen und Krimisubgenres erarbeitet werden, ohne sich dezidiert auf den deutschsprachigen Provinzkrimi zu beziehen, wird das Motiv der Nutrition auf der Ebene akzidenteller Narrationsbausteine verortet. Zum einen wird es als Auslöser textexterner Effekte, wie die Bewerbung der beschriebenen Region als touristische Attraktion, klassifiziert. Zum anderen wird ihm in textinterner Hinsicht die Rolle eines Nebenmotivs, das an lateralen Erzählsträngen mehr oder weniger ornativ beteiligt ist, zugeschrieben, z. B. Kochen als stressabbauendes Hobby der Ermittlerfigur ${ }^{3}$. Im Gegensatz zu diesem als dekoratives Accessoire beschreibbaren Status ist im süddeutschen Provinzkrimi die Funktion des Nutritionsdiskurses substanzieller Art. Als Teil des Ensembles an Strategien zur narrativen Konstruktion von Provinz hat er genrekonstitutive Funktion, da die - systematisch noch zu beschreibende - Gattung des Provinzkrimis, wie z.B. der Wiener Krimi zu den durch das Motiv des Raums profilierten Krimisubgenres gehörend, essentiell durch das Erzählen von Provinz bestimmt wird. An den Erfolgsserien von Volker Klüpfel / Michael Kobr, Rita Falk und Jörg Maurer sollen die für diese Gruppe von Provinzkrimis spezifische Formation des Ernährungsdiskurses beschrieben und deren Leistung für die Konturierung des literarischen Raumes Provinz herausgearbeitet werden, wobei aus Platzgründen unter Ausklammerung des Aspekts des Trinkens der Fokus auf dem Essen liegt.

\section{Orgien der Gaumenfreuden - der Speiseplan im Provinzkrimi}

Träger des Ernährungsdiskurses sind in den Serien von Klüpfel / Kobr sowie Rita Falk die Protagonisten, die Ermittler Kluftinger und

Zum Kriminalroman der Gegenwart, Bozen, Innsbruck, München u.a., StudienVerlag 2004 (Schriftenreihe Literatur des Instituts für Österreichkunde; Bd. 15), 121-143. Die Diplomarbeit von Brigitta Eva Gruber mit dem Titel Heiß serviert - kalt gemacht. Kulinarik im deutschsprachigen Kriminalroman (Univ. Wien 2013) gehört zwar auch zu dieser Kategorie, vermittelt jedoch keine weiterführenden Informationen.

2. - Vgl. z.B. Katrin Giritzhofer: Mörderisch und Kulinarisch: Eva Rossmanns Frauenduo Mira und Vesna zwischen Wien, Wein und Veneto, Dipl.-Arb. Univ. Wien 2008; Thomas Kniesche: „Gärgas: Die Kriminalromane von Alfred Komarek“, in: The German Quarterly, 2006, 79, 2, 211-233; Mediha Ljaic: Die Eberhofer-Krimis von Rita Falk, Dipl.-Arb. Univ. Wien 2014.

3. - Vgl. Strigl: „Hedonismus“ u. Griem: „Mordshunger“, a.a.O., passim. 
Franz Eberhofer, in Jörg Maurers Krimis dagegen zwei Nebenfiguren, das zur kriminellen Abteilung des Personals gehörende Bestatterehepaar Grasegger. Anstatt entlang der Präsenz der Zentralgestalt die gesamte Erzählung zu durchziehen, tritt hier die Nahrungsthematik, ohne in der restlichen Handlung eine Rolle zu spielen, in den Grasegger-Szenen komprimiert auf. Diese beiden Personen firmieren gleichsam als Personifikationen des Essens.

Generell kann statuiert werden, dass im Provinzkrimi überdurchschnittlich oft, viel und reichhaltig gegessen wird. Die Eberhofer-Serie besetzt Platz eins, was die quantitative Evidenz des Alimentationsdiskurses betrifft, da im Mittelwert auf jeder dritten bis vierten Seite vom Essen die Rede ist. Die dreimal täglich stattfindenden Hauptmahlzeiten, begleitet von an Anzahl variierenden Zwischenmahlzeiten, haben geradezu leitmotivischen Charakter und versorgen die Handlung mit einer gleichmäßigen kulinarischen Grundierung, was das Thema Essen zum Gerüst werden lässt, um das herum sich alles Weitere gruppiert und gleichsam die übrigen Elemente im Leben der Figuren zu fakultativen Zutaten von nicht annähernd großer Bedeutung degradiert. Bei Kluftinger ist die Frequenz der Beschreibungen von Nahrungsaufnahme weniger hoch, die einzelnen Ausführungen jedoch detaillierter und einlässlicher. Was die Graseggers betrifft, scheint in ihrer Existenz für alles andere außer Essen nur schwer ein Platz zu finden zu sein. Das Essen beginnt bei Tagesanbruch, während einer Mahlzeit wird bereits die nächste geplant, und die Planung einer nichtalimentären Aktivität ist unweigerlich an die Überlegung geknüpft, wie dies zeitlich mit der Einhaltung der nächsten Mahlzeit zu vereinbaren wäre 4 .

Der alltägliche Speiseplan von Eberhofer, Kluftinger und dem Ehepaar Grasegger präsentiert sich als wahres Defilee opulentester Gerichte: Kässpatzen, Schweinshaxe mit Sauerkraut und Knödel, Schweinsbraten mit Sauerkraut und Knödel, Krustenbraten mit Krautsalat und Knödel, Zigeunerschnitzel mit Bratkartoffeln und Gurkensalat, Jägerbraten mit Pilzrahmsauce, Reis und Salat, Rindsrouladen, gespickter Rehrücken, Rahmgulasch mit Spätzle, Buttergemüse und Preiselbeerkompott, „Linsen mit Spätzle und Ripple“5, Dampfnudeln mit Vanillesauce, Marillenknödel mit Butterbrösel, Zwetschkendatschi mit Butterstreuseln und Mandeln, garniert mit Schlagsahne, Gamsfiletsulz, ,Knöcherlsülze“, „Regensburger Wurstsalat" und paniertes „Wammerl“6, eine 303.

4. - Vgl. Jörg Maurer: Unterholz. Alpenkrimi, Frankf. / Main, Fischer 2014 [U],

5. - Volker Klüpfel, Michael Kobr: Seegrund. Kluftingers dritter Fall, 8. Aufl., München, Piper 2009 [S], 179.

6. - Jörg Maurer: Föhnlage. Alpenkrimi, Frankf. / Main, Fischer 2013, 93. 
Aufzählung, die sich beinahe unbegrenzt fortsetzen ließe $\mathrm{e}^{7}$. Mit diesen lukullischen Mählern noch nicht genug, lassen sich die Figuren mindestens einmal pro Tag, gern jedoch öfter, eine „Brotzeit"8 schmecken, die ihrerseits nicht Schmalhans zum Küchenmeister hatte. Ein solcher Imbiss Kluftingers, worin ihm Eberhofer und mehr noch Graseggers in nichts nachstehen, setzt sich im Durchschnitt folgender Art zusammen:

Ein Paar Schüblinge mit Semmel, ein Paar Landjäger mit Semmel, Senf für die Schüblinge und die Landjäger, eine Semmel mit geräuchertem Schinken und ein mit einer eineinhalb Zentimeter dicken Schicht aus Bauernsalami belegtes Brot (mit der Wurst sollte man beim belegten Bort nie sparen, das machten seiner Ansicht nach die meisten Menschen falsch), zwei Käsesemmeln, falls er keine Lust auf Wurst haben sollte, und außerdem, weil Käse den Magen schließt, eine Banane, um etwas Leichtes für zwischendurch zu haben, und eine Tafel Schokolade für den Nachtisch ${ }^{9}$.

Der sich dieserart als Prozession wahrer Orgien der Gaumenfreuden gestaltende Ernährungsdiskurs des Provinzkrimis steht quer zu zwei bestimmenden Komponenten des aktuellen Ernährungsdiskurses der außerfiktionalen Realität. Diese können beschrieben werden als diätetischer und multikulturalistischer Subdiskurs, ersterer gegenwärtig hochbrisant und das allgemeine Ernährungsverhalten revolutionierend, zweiterer ein etabliertes und damit selbstverständliches Essverhalten bezeichnend.

\section{3. „Die lassen sichs schmecken, und sie stehen dazu“10_ gegen den diätetischen Ernährungsdiskurs}

Mit dem Begriff des diätetischen Diskurses wird der unter dem Primat des Gesundheitsbewusstseins stehende Blick auf die Nahrung bezeichnet, an dem die Inversion des traditionellen Konzepts von Essen beobachtbar ist. Die elementare Qualität von Nahrung als unmittelbarster Lebensspender und ihre damit verbundenen essentiellen Parameter, Stillung des Hungers und Schaffung von Genuss, erfahren eine Verkehrung in ihr Gegenteil. Der diätetische Diskurs konzeptualisiert Nahrungsmittel statt als unverzichtbar daseinserhaltend als potentielle Bedrohung für den Körper und damit das Leben, was eine essayistische Reaktion in der aktuellen Presse mit dem Titel „Der Feind

7. - Vgl. die genannten Serien passim.

8. - Volker Klüpfel, Michael Kobr: Milchgeld. Kluftingers erster Fall, 7. Aufl., München, Piper 2008 [M], 253.

9. - Ebd.

10. - Maurer: U, a.a.O., 57. 
auf meinem Teller"11 griffig formuliert. Das Essen, die wohl ursprünglichste der anthropologischen Konstanten, ist in Misskredit geraten, es wird auf allerorts lauernde Gefahr hin beargwöhnt, als Quell jeglichen Übels überhaupt diskreditiert und aus diesem Dispositiv heraus als der Ansatzpunkt gesehen, solches zu bekämpfen. Dies beginnt mit einer bestimmten Richtlinien folgenden peniblen Auswahl der Lebensmittel, etwa dem Verzehr ausschließlich mit dem Prädikat ,Bio ${ }^{6}$ zertifizierter Lebensmittel, setzt sich fort über den Verzicht auf ganze Lebensmittelgruppen, die in den Geruch des Schädlichen geraten sind, und gipfelt in der akribischen, meist alternativmedizinisch betriebenen Fahndung nach möglichen Allergien, Intoleranzen und Unverträglichkeiten, unabhängig davon, ob sich diesbezügliche Symptome gezeigt haben oder nicht. Beispielsweise glauben laut einer Umfrage der NDP Group aus dem Jahr 2013 in den USA 41 Prozent der erwachsenen Bevölkerung, glutenfreie Ernährung sei auch für Menschen angezeigt, die nicht an Zöliakie leiden ${ }^{12}$.

Diente Essen in seiner Funktion als Mittel gegen den Hunger traditionell unumstritten und vorrangig dem Zweck, den Organismus mit Nahrung zwecks Aufrechterhaltung des Stoffwechsels zu versorgen, so ist ein wesentlicher Aspekt des diätetischen Diskurses darauf gerichtet, dem Körper Nahrung zukommen zu lassen, die ihn nicht ernährt. Als darauf zielende Anstrengung ist jedenfalls das stetig wachsende Angebot eindringlich beworbener Diäten, wie der populären Atkins-, Detox- oder Volumetrics-Diät, zu bewerten, die sämtlich zum primären Ziel haben, Zufuhr von Nährstoffen sinnlich vorzutäuschen, ohne dies physiologisch zu realisieren oder, verschärft, den Körper zum Abbau von Nährstoffreserven zu zwingen.

In der diskursarchitektonischen Logik erscheint es nur folgerichtig, dass eine in diesem Ausmaß dämonisierte, geradezu zur Büchse der Pandora geratene Kategorie als Lieferantin von Genuss nicht mehr in Frage kommt. Etwas, vor dem man sich unausgesetzt fürchten und schützen muss, kann für die freudvolle Kulturpraktik des Genießens nicht zur Verfügung stehen, sondern muss möglichst reduziert werden. Das in dem diätetischen Diskurs installierte Äquivalent der traditionellen Kategorie Genuss ist der Verzicht. ,[D]ieser Ernährungszeitgeist, bei dem man immer irgendwas nicht mehr isst, dessen wichtigstes Genusswort ein riesiges OHNE ist" 13 , bringt interessanterweise eine Rhetorik hervor, die formal in die Position des Lobs der Speise eintritt,

11. - Martin Dworschak: „Der Feind auf meinem Teller“, in: Der Spiegel, 2015, 7, 104-108, hier 104.

12. - Vgl. ebd., 105.

13. - Jochen-Martin Gutsch: „Vegane Grillschnecken“, in: Der Spiegel, 2015, $34,59$. 
dessen Inhalt jedoch invertiert: anstatt den Wohlgeschmack des Mahls zu rühmen, also das Vorhandensein von genussstiftenden Faktoren - wie z.B. Würze, dass es eine Fleischspeise ist, bevorzugte Beilagen - positiv zu bewerten, wird ausgedrückt, die Absenz solcher nicht zu beklagen. Wie ein Essayist des Spiegels eine solche Sprechsituation humorvoll imaginiert: „Die Simone und ich - wir essen jetzt keinen Weizen mehr. Und wir vermissen nichts!" 14

Die Kategorie des bloßen Food Trends ${ }^{15}$ bereits weit hinter sich gelassen habend, kann dem diätetischen Diskurs wohl die Qualität eines ideologieähnlichen Systems attestiert werden, dessen konstitutiver Wert die Herstellung oder Wahrung eines mit dem Begriff der ,Gesundheit' umschriebenen Zustandes ist. Aus seiner diskursinternen Funktionalisierung als Containerbegriff für die Erfüllung beliebiger Sehnsüchte und Heilsversprechen bezieht diese Vokabel eine Bedeutung, die über die Abwesenheit pathologischer Phänomene weit hinausragt und hochgradig moralisch aufgeladen ist. In diesen Kontext gestellt, mutiert Nahrung zu dem Instrument der Erlangung dieses Zustandes und die darauf ausgerichtete Optimierung der Praktiken ihrer Aufnahme zum kategorischen Imperativ der Ernährungsideologie. Dieserart metaphysisch legitimiert, gewinnt der diätetische Diskurs normative Kraft, die es vermag, als Mechanismus gesellschaftlicher Ein- und Ausgrenzung wirksam und zum Distinktionskriterium von Wohl- und Fehlverhalten zu werden. Klotter konstatiert, „,[w]er sich entsprechend der offiziellen Doktrin dem Anschein nach nicht gesundheitsgerecht verhält, darf [...] massiv diskriminiert und stigmatisiert werden" 16 . Der soziale Druck, der durch die alimentäre ,In-Group" auf die ,Out-Group' ausgeübt wird, artikuliert sich im Eindringen von binär bewertenden Oppositionsbildungen wie „Seht her, ein kultivierter Esser, keiner von den wahllos schaufelnden Allesvertilgern" 17 in den Alltagsdiskurs und in der Infiltration der Selbstwahrnehmung der Nonkonformen durch die Perspektive der Gegner, sich manifestierend in Aussagen, man fühle sich ,wie der letzte Allesfresser"18. Für die Umsetzung des diätetischen Diskurses in extremer Ausprägung wurde

14. - Ebd.

15. - Vgl. Wolfgang Reiter, Hanni Rützler: „Vorwärts zum Ursprung. Gesellschaftliche Megatrends und ihre Auswirkungen auf eine Veränderung unserer Esskulturen“, in: Angelika Ploeger, Gunther Hirschfelder, Gesa Schönberger (Hg.): Die Zukunft auf dem Tisch. Analysen, Trends und Perspektiven der Ernährung von morgen, Wiesbaden, Springer 2011, DOI: 10.1007/978-3-531-93268-2, 77-88, hier 81-84.

16. - Christoph Klotter: Fragmente einer Sprache des Essens. Ein Rundgang durch eine essgestörte Gesellschaft, Wiesbaden, Springer 2015, DOI: 10.1007/978-3658-07065-6, 96.

17. - Dworschak: „Feind“, a.a.O., 105.

18. - Gutsch: „Grillschnecken“, a.a.O. 
bereits der medizinische Terminus „Orthorexia nervosa“ 19 geprägt, der die Klassifikation solchen Verhaltens, obzwar noch nicht offiziell als Krankheit anerkannt, als „Essstörung, die darin besteht, sich übermäßig und zwanghaft gesund zu ernähren“"20, vornimmt.

In den Provinzkrimis wird mittels mehrerer poetologischer Strategien in verschiedenen Graden gegen den diätetischen Diskurs angeschrieben. Zum einen wird schlichtweg seine Absenz vorgeführt. Es wird ein Panorama gestaltet, in der ein von der oben beschriebenen Kategorieninversion gänzlich unbetroffenes Ernährungsverhalten besteht. Das Vertrauen in die Nahrung ist ungebrochen, sie wird als in jeder Hinsicht lebensnotwendige und -freundliche und damit naturgemäß positive Instanz konzeptualisiert. Hunger und Appetit wirken ohne jedwede Modifikation durch diätetische Parameter als unmittelbare Initiatoren für Nahrungsaufnahme. „Als er [Kluftinger] im Auto saß, beschlich ihn ein leises Hungergefühl, das sich auf dem Weg nach Kempten zu einem ordinären Kohldampf ausgeweitet hatte. Wie ferngesteuert bog Kluftinger zum Eisstadion ab und hielt vor einem Imbisswagen [...], für ihn zählte jetzt nur die schnelle Nahrungsaufnahme“21, und genauso für Eberhofer: „Da es jetzt schon auf Mittag zugeht und mich naturgemäß sakrisch der Hunger packt, fahr ich erst einmal heim“22. Das dringende physiologische Verlangen nach Nahrung besitzt sogar eine Macht eines Ausmaßes über die beiden, das sie jede Sparsamkeit vergessen lässt. Kluftinger will nach einer kleineren Bergwanderung seine Familie ganz gegen seine Gewohnheit in ein Lokal einladen, ,wobei seine Großzügigkeit von der Tatsache befördert wurde, dass sich sein Appetit inzwischen zu einem quälenden Hungergefühl ausgewachsen hatte" 23 . Eberhofer plündert die Dorfmetzgerei: „Derweil kauf ich ein paar Angebote, weil ich schon mal da bin. Weil ich schon mal da bin und der Hunger die Auslage der Metzgerei zu einem wahren Schlaraffenland macht" 24 . Es kann kein Zweifel bestehen, dass das Ziel nutritiver Akte die Zufuhr nahrhafter Nahrung ist. In Kluftingers Reflexionen betreffend das Verhältnis von Hunger und Essen wird dies explizit:

Er hatte das Gefühl, ein großes Loch in seinem Magen zu haben. Das war immer so, wenn er am Vorabend nicht genügend gegessen hatte. Da half auch ein ausgiebiges Frühstück nichts mehr. Aus seiner Sicht verhielt es sich mit Nahrung wie mit Schlaf: Ein einmal erlittenes Defizit war so schnell nicht mehr aufzuholen. Man schleppte es immer

19. - Klotter: Fragmente, a.a.O., 96.

20. - Ebd., vgl. 98.

21. - Klüpfel, Kobr: M, a.a.O., 54.

22. - Rita Falk: Dampfnudelblues. Ein Provinzkrimi, München, dtv 2011 [D], 34.

23. - Klüpfel, Kobr: S, a.a.O., 13.

24. - Falk: D, a.a.O., 193. 
ein Weilchen mit sich herum. Und die paar verkohlten Fischstäbchen und die welken Blättchen Salat, die ihm beim Grillen gestern geblieben waren, hatten seinen Nährstoffhaushalt eindeutig ins Minus getrieben ${ }^{25}$.

Anstelle des Praktizierens von Diäten wird hemmungslose Völlerei erzählt, deren ins Hyperbolische reichende Gestaltung bereits in eine über die Präsentation der Diätabsenz hinausgehende dezidierte Verhöhnung des diätetischen Diskurses hineinreicht. So ordert Kluftinger im Restaurant Zwiebelrostbraten mit Käsespätzle ${ }^{26}$, macht also eine weitere Hauptspeise zur Beilage, und verzehrt seinen „Zwetschkendatschi“ in der Zusammensetzung „,ein Teil Kuchen, drei Teile Sahne“27. Eberhofer, von seinem Freund Birkenberger eingeladen, bestellt und isst ,alles, was auf der rechten Seite [der Speisekarte] steht“"28, und Graseggers „,stellten ein nahrhaftes Frühstück auf den Tisch der Terrasse, das für Jesus und die zwölf Jünger gereicht hätte“29.

Das Auswahlverfahren der Nahrungsmittel und die Regulation der Nahrungsmenge werden durch Vorlieben und Appetit gesteuert, gesundheitliche oder körpergewichtsbezogene Kategorien kommen in den überaus seltenen Fällen ihres Auftretens dafür nicht in Betracht. So lässt sich Kluftinger durch offensichtliche Unverträglichkeit nicht am Konsum seiner Lieblingsspeise, der „Kässpatzen“, hindern: „Er schlug ja auch jedes Mal kräftig zu, obwohl ihm bewusst war, dass ihm so fette Speisen eigentlich nicht bekamen. Er wusste, dass ihn heute Nacht sein Sodbrennen wieder heimsuchen würde, die vielen in Butter gebräunten Zwiebeln würden schon dafür sorgen. Und doch liebte er diese deftige Kost. Besonders die Zwiebeln“30. Ebenso wenig veranlassen ihn seine „,schätzungsweise fünfzehn Kilo Übergewicht“31, auf den Verzehr eines fünften Krautkrapfens zu verzichten, denn ,den aus dünnem Nudelteig gerollten, mit Speck und Sauerkraut gefüllten Rollen konnte er einfach nicht widerstehen. [...] Und er hatte auch nichts dagegen einzuwenden, wenn Butter oder Schmalz oder am besten Butterschmalz aus ihnen rann“32. Eberhofer zeigt beim Einkauf von Leberkäsesemmeln ein adäquates Verhalten: „,Drei oder vier?‘, fragt er [der Metzger] und

25. - Volker Klüpfel, Michael Kobr: Laienspiel. Kluftingers vierter Fall, 2. Aufl., München, Piper 2009, 227.

26. - Dies.: Erntedank. Kluftingers zweiter Fall, 18. Aufl., München, Piper 2010 [E], 168.

27. - Ebd., 236.

28. - Rita Falk: Schweinskopf al dente. Ein Provinzkrimi, München, dtv 2013 [SD], 101.

29. - Maurer: U, a.a.O., 60.

30. - Klüpfel, Kobr: M, a.a.O., 5.

31. - Ebd., 201.

32. - Klüpfel, Kobr: S, a.a.O., 254. 
öffnet die heiße Vitrine. ,Zwei', sag ich und greif körpermittig nach dem Winterspeck, der sich dort in den letzten Wochen angesammelt hat. Die Leberkäswolke findet auf Anhieb den Weg direkt in meine Nasenlöcher. Mir trieft der Zahn. ,Vier', sag ich. ,Mach vier, Simmerl!““33 Das traditionelle Verhältnis zur Nahrung, das Klotter mit den Worten „Ich esse, was mir schmeckt, und was ich überhaupt bekomme" beschreibt und das den polaren Gegensatz zu dem diätetischen „Ich muss die Inhaltsstoffe zu mir nehmen, die gesund sind“"34, darstellt, ist im Universum des Provinzkrimis noch in voller Gültigkeit. Die wohl hohnvollste Absage wird dem diätetischen Diskurs durch die Haltung des Bestatterehepaars erteilt, in der durch ihn als Feindbilder verdammte Kategorien statt rejiziert gefeiert werden: „Ursel Grasegger [...] hatte wie Ignaz, ihr Gemahl, einen breiten Fünfzigpfünder-Gürtel um den Leib angesetzt. Beide trugen die Speckschwarten wie Königsmäntel, und man wusste sofort: Die lassen sichs schmecken, und sie stehen dazu“35.

Ebenso wie die Bedeutung des Hungers ist auch die des Genusses unverändert. Essen hat einen überaus hohen lustgenerierenden Stellenwert. Die Einverleibung der beschriebenen Köstlichkeiten bedeutet für Eberhofer, Kluftinger und die Bestatter ultimativen Genuss, einen unverzichtbaren Faktor der Lebensqualität, die kulinarische Befriedigung ist Voraussetzung für physisches und psychisches Wohlbefinden. Eberhofer wird durch eine von „,der Oma“"36 liebevoll zubereitete, üppige Mahlzeit mit einem frustrierenden Arbeitstag versöhnt und umgekehrt beeinträchtigt es seine Stimmung immens, wenn keine solche für ihn bereitsteht: „Wie ich mittags daheim zur Tür reinkomm: ein Albtraum allererster Klasse. Kein würziger Essensduft im Hausgang, kein zischendes Brutzeln in den Pfannen, kein Geschirrklappern. Gar nichts“37. Für Kluftinger sind die „Kässpatzen [...] das Beste am Montag, das Einzige, weswegen er ihn überhaupt ertragen konnte“38. Und „Ursel leckte den Teller mit der Gamsfiletsülze aus" 39 , was deutlich macht, wie gut es ihr geschmeckt hat. Gleichermaßen herrscht im Provinzkrimi die traditionelle genussverherrlichende Rhetorik. Eberhofer äußert sich über die Speisen im Allgemeinen, insbesondere aber über die Kochkünste seiner Großmutter stets in geradezu hymnischer Euphorie. „Der Obatzte

33. - Falk: D, a.a.O., 10.

34. - Klotter: Fragmente, 98.

35. - Maurer: U, a.a.O., 57.

36. - Rita Falk: Winterkartoffelknödel. Ein Provinzkrimi, 7. Aufl., München, dtv 2012 [W], 13.

37. - Falk: D, a.a.O., 8 .

38. - Klüpfel, Kobr: M, a.a.O., 5.

39. - Maurer: U, a.a.O., 82. 
ist ein Gedicht“40, die Brotzeit ist ,göttlich“41, ,,[d]ie Oma hat ein hammermäßiges Frühstück gezaubert" ${ }^{42}$, sogar das von ihm selbst nach dem Rezept der Oma hergestellte „Essen ist einfach phänomenal“43, der „Apfelstrudel ist ein zur Materie gewordener Traum“44, ,[w]as aber der absolute Hammer ist und dir wirklich den Zahn triefen lässt, das ist die Soße von der Oma. Schmeckt einfach zum Reinknien, ehrlich“45, und „dazu gibt's einen Kartoffelsalat, in dem ich gern ersaufen möchte" ${ }^{\text {“46 }}$. Der aus dem Verzehr von Weißwurst gezogene Lustgewinn wird gar zu dem Effekt von Rauschmittelgenuss vergleichbarer Ekstase überhöht: Ursel Grasegger ,schloss die Augen und lehnte sich zurück, als ob sie an einem Joint gezogen hätte" 47 . Weniger überschwänglich artikuliert Kluftinger seine Begeisterung für das Essen, doch auch er spricht z. B. von dem „geliebten Zwiebelrostbraten mit Kässpatzen“48. Aussagen über den Verzicht drücken keineswegs gesundheitsbewegte Billigung aus, sondern höchstes Missfallen. Eberhofer lamentiert angesichts einer fleischlosen Mahlzeit: „Daheim gibt's Salzkartoffeln, Spinat und Spiegeleier. Wie gesagt, die Fastenzeit ist ein Martyrium hier bei uns“49.

Eine Steigerungsstufe über der Absenz des diätetischen Diskurses steht seine Diskreditierung durch Zuordnung zu narrationsinternen Antipathieträgern oder als lächerlich gezeichneten Figuren. Der erklärte Feind Kluftingers, der von ihm als verabscheuungswürdig befundene Dorfarzt Martin Langhammer ist ein glühender Verfechter der Ernährungsreformen und Eberhofers Freund Rudi Birkenberger, der in seinen Augen trotz Zuneigung in weltanschaulicher Hinsicht nicht ernst zu nehmen ist, lässt sich immer wieder dazu hinreißen, aktuelle Modeerscheinungen mitzumachen, weil er es für chic hält, darunter auch die diätetische Strömung, im Zuge dessen er grünen ,Smoothie“ frühstückt (siehe unten). Der Nahrungsmittelvorrat Langhammers ist bezeichnend und weist ihn als wahren Guru der diätetischen Ideologie aus. Auf seiner verzweifelten Suche nach etwas Essbarem anlässlich eines Übernachtungsaufenthaltes im Haus des Arztehepaars stößt Kluftinger in der Küche nur auf „diverse Tupperdosen mit verschie-

\footnotetext{
40. - Falk: D, a.a.O., 80.

41. - Ebd., 95.

42. - Falk: SD, a.a.O., 78.

43. - Dies.: S, a.a.O., 160.

44. - Dies.: Griessnockerlaffäre. Ein Provinzkrimi, München, dtv 2012 [G], 77.

45. - Dies: Zwetschgendatschikomplott. Ein Provinzkrimi, München, dtv 2015

46. - Dies.: G, a.a.O., 80.

47. - Jörg Maurer: Oberwasser. Alpenkrimi, Frankf. / Main, Fischer 2013, 179.

48. - Volker Klüpfel, Michael Kobr: Herzblut. Kluftingers neuer Fall, Augsburg, Weltbild 2013, 136.

49. - Falk: SD, a.a.O., 36.
} [Z], 175 . 
denfarbigen Körnern und Flocken [...], dann auf schätzungsweise dreißig verschiedene Teesorten“, im Kühlschrank präsentiert sich das entsprechende Tableau: „Kein Gramm Wurst lag in den akribisch geordneten Fächern. Stattdessen standen in der obersten Etage eine Menge kleiner Joghurtfläschchen, die nicht etwa ,Erdbeere' oder ,Banane' hießen, sondern mit dem kryptischen Kürzel ,LC I!' versehen waren. [...] Saftflaschen lagen in der untersten Reihe und aus dem Gemüsefach schaute eine Menge Grünzeug heraus" ${ }^{50}$.

Als Krönung dieser impliziten Diffamierungsstrategien wird ein Frontalangriff gegen jene zwei Nahrungsmittel geführt, die den wohl ausgeprägtesten Symbolcharakter für die moderne diätetische Essensordnung haben, das Müsli und den ,Smoothie‘. Beide werden mit einer Semantik belegt, die sie als nicht für den menschlichen Genuss geeignete Substanzen kennzeichnen. Kluftinger betrachtet das von Doktor Langhammer mit einem typischen Schlagwort des diätetischen Diskurses als die „reinste [...] Powernahrung“51 angepriesene Müsli als ,eine Schüssel Vogelfutter“52, Eberhofer sieht Birkenberger bei der Zubereitung des grünen Smoothies ,mit irgendwelchen Blumensträußen hantieren" 53 , zunächst ohne zu erkennen, dass es sich um Kräuter handelt. Darüber hinaus werden beide Diätprodukte als übelschmeckend bis ekelerregend deklariert. Das Müsli verbreitet in Kluftingers Mund mehligen Geschmack und „die Körner schienen ihn aus ihrem Sojabad grau-bräunlich anzuglotzen. Er bekam eine Gänsehaut" 54 , der Smoothie präsentiert sich als „,kotzgrüne, zähflüssige Masse“ ${ }^{\text {55 }}$. Beide Male wird die Aufnahme dieser Nahrung verweigert. Kluftinger „konnte die Schüssel unmöglich auslöffeln, das war ihm klar. Sein Magen knurrte, aber es ging einfach nicht" 56 . Deutlich radikaler, lässt sich Eberhofer auf einen Versuch gar nicht erst ein: „Ich kipp mein Glas in den Ausguss und setz stattdessen Kaffee auf" 57 . Mehr noch, die für ,Gesundheit" stehenden Lebensmittel sind nicht nur von abstoßendem Geschmack, sondern erzeugen pathologische Symptome. Der auf Langhammers Geheiß genossene Vollkornkuchen bringt „Kluftingers Verdauung so nachhaltig durcheinander [...], dass er am Abend mehrmals das Wohnzimmer verlassen musste, um seine Blähungen loszuwerden" 58 . Als Birkenberger infolge des Schmoothiegenusses von akuter Diarrhoe überfallen wird,

\footnotetext{
50. - Klüpfel, Kobr: E, a.a.O., 44.

51. - Ebd., 47.

52. - Ebd., 88.

53. - Falk: Z, a.a.O., 65.

54. - Klüpfel, Kobr: E, a.a.O., 47.

55. - Falk: Z, a.a.O., 66.

56. - Klüpfel, Kobr: E, a.a.O., 47.

57. - Falk: Z, a.a.O., 66.

58. - Klüpfel, Kobr: E, a.a.O., 319.
} 
wird der Höhepunkt der Polemik gegen den diätetischen Diskurses erreicht: sogar sein Repräsentant wird durch ihn geschädigt.

\section{4. „Da scheiß ich auf eine jede Paella! ${ }^{659}$ - gegen den multikulturalistischen Ernährungsdiskurs}

In der außerfiktionalen Realität der Gegenwart gilt eine multikulturalistische Ernährungsweise keineswegs mehr als außergewöhnlich und die Küche bestimmter Kulturkreise, an erster Stelle wohl Italienisch und Chinesisch / Japanisch, hat schon länger Einzug in den Ernährungsstandard gehalten. Zwischen überlieferter einheimischer und mittlerweile etablierter fremdländischer Kulinarik herrscht die konzeptionelle Differenzierung von ,alt' und ,neu'. Es ,erscheint das ,Fremde längst vereinnahmt als das ,Neue“" 60 , das Fremde hat Integration in den Kanon des Akzeptierten in der Position des Neuen gefunden.

Im Provinzkrimi dagegen ist interkulturelle Kochkunst weder etabliert noch akzeptiert. Abgesehen davon, dass überregionale Gerichte am regulären Speiseplan nicht vorkommen, werden sie in den seltenen Fällen der Konfrontation der diskurstragenden Figuren mit solchen kompromisslos abgelehnt. Dies erfolgt durch dem antidiätetischen Diskurs analoge Mechanismen. Zum einen treten wiederum die Antipathieträger als Repräsentanten des multikulturalistischen Diskurses auf, gepaart mit seiner Zurückweisung durch die Träger des Antidiskurses. Das illustrativste Beispiel für diese Formation ist wohl in der unmittelbaren Gegenüberstellung der entgegengesetzten Positionen zu finden: „,Muscheln, herrlich!', jubilierte Langhammer, und Kluftinger griff sich resigniert ein Stück Brot aus dem Korb. [...] Niemals würde er dieses Zeug anrühren [...]. Muscheln und Kaviar in den Allgäuer Bergen - Mahlzeit" 61 . Zum anderen werden die multikulturellen Speisen als unwillkommen, schlecht schmeckend, ekelerregend bis toxisch deklariert und ihr Konsum zumeist boykottiert. Die Unerwünschtheit demonstriert Eberhofer sehr plakativ, wenn er sich, eben von einem Mallorca-Aufenthalt zurückgekehrt, mit einer stoßseufzerähnlichen Gesinnungsäußerung von der fremdkulturellen Küche distanziert: „Wie wir heimkommen, hat die Oma gekocht, das kann man gar nicht glauben. Mit einer Tomatensuppe als Vorspeis und einem Apfelkuchen als Nachspeis. Dazwischen ein Kalbsragout mit Spätzle

59. - Falk: W, a.a.O., 178.

60. - Ulrich Tolksdorf: „Das Eigene und das Fremde. Küchen und Kulturen im Kontakt", in: Alois Wierlacher, Gerhard Neumann, Hans Jürgen Teuteberg (Hg.): Kulturthema Essen. Ansichten und Problemfelder, Berlin, Akademie Verlag 1993 (Kulturthema Essen; Bd. 1.), 188-192, hier 188.

61. - Volker Klüpfel, Michael Kobr: Rauhnacht. Kluftingers fünfter Fall, 3. Aufl., München, Piper 2010, 56. 
und Preiselbeeren. Da scheiß ich auf eine jede Paella!"62 Kluftinger, der aus Versehen in einem Restaurant ,Kässpatzen ungarischer Art“ bestellt, erblickt darin, dass ,jemand auf die aberwitzige Idee kommen konnte, Kässpatzen mit Gulasch oder Letscho zu kreuzen“, eine „kulinarische [...] Vergewaltigung seiner Leibspeise"63, wobei er die Erfahrung macht, „,dass der Grundsatz seines Vaters in solchen Situationen - ,Der Hunger treibt's nei!' - nicht auf jede beliebige Konstellation von Lebensmitteln anwendbar war"64. Graseggers leiden zu wesentlichen Teilen aus kulinarischen Gründen schwer unter der zwecks Flucht vor dem Arm des Gesetzes selbstgewählten Verbannung aus der Werdenfelser Heimat in das „,italienische [...] Exil“65. An anderer Stelle löst die Begegnung mit exotischen Nahrungsmitteln alle psychophysischen Reaktionen des Ekels aus. Angesichts von Muscheln und Tintenfisch in seiner heimischen Küche „bekam [Kluftinger] eine Gänsehaut“ und zeigt intensive Berührungsängste, da er nur „mit spitzen Fingern die Tüte“ anfasst und dabei „den Kopf in eine andere Richtung " dreht ${ }^{66}$. Eberhofer verweigert den Verzehr englischer Lammkoteletts in Minzsoße mit der Begründung: ,es ist einfach ekelhaft. Ekelhaft und völlig ungenießbar"67. Der Diätnahrung entsprechend sind die multikulturellen Gerichte nicht nur als Genuss-, sondern auch als Lebensmittel ungeeignet, denn sie tragen nicht, wie es die Aufgabe solcher wäre, zur Kräftigung des Organismus bei, sondern rufen gastrointestinale Beschwerden hervor. Nach einem Besuch im Sushilokal, einer plötzlichen und im Nachhinein sofort bereuten Anwandlung kultureller Aufgeschlossenheit Kluftingers geschuldet, ,hatte er nichts gefrühstückt, weil es seit dem unfreiwilligen Fischessen am gestrigen Abend in seinem Magen gärte" 68 .

Ein symbolischer Akt der Verbannung fremdkultureller Kulinarik aus der Küche der Provinz wird in der Eberhofer-Serie gesetzt. Das singuläre Eindringen eines nichtregionalen Gerichts, das bereits erwähnte Lammfleisch mit Minze, verursacht durch ein als Geschenk erhaltenes Kochbuch mit internationalen Rezepten, wird durch unmittelbare Entsorgung des Essens und des Buchs sofort wieder rückgängig gemacht und als inakzeptabel verworfen. „Ich kann's beim besten Willen nicht essen, geh rüber zum Mülleimer und kipp das Zeug weg. Der Papa tut's mir gleich. Und obwohl die Oma ansonsten sehr empfindlich ist,

62. - Falk: W, a.a.O., 178.

63. - Klüpfel, Kobr: S, a.a.O., 62.

64. - Ebd., 63.

65. - Jörg Maurer: Niedertracht. Alpenkrimi, Frankf. / Main, Fischer 2012, 148, vgl. $150-151$.

66. - Klüpfel, Kobr: M, a.a.O., 87.

67. - Falk: SD, a.a.O., 20.

68. - Klüpfel, Kobr: S, a.a.O., 94. 
was ihre Kochkunst betrifft, folgt auch ihr Tellerinhalt prompt dem unseren. Das neue Kochbuch fliegt gleich hinterher"69.

\section{Die Konstruktion von Provinz}

Der Alimentationsdiskurs der hier untersuchten Gruppe von Provinzkrimis vertritt die radikale Opposition zu zwei bestimmenden Komponenten des außerfiktionalen Ernährungsdiskurses, der diätetisch und der interkulturell orientierten Nahrungsorganisation. Jene Instanzen, die die narrationsinterne Deutungshoheit von Welt innehaben, lehnen das diesen Diskursen zugeordnete Speisenrepertoire durch die Bewertung als geschmacklich schlecht und unbekömmlich sowie die Verweigerung seiner Aufnahme radikal ab, was einem objektiven Verdikt, der Exklusion aus dem kulturspezifischen Katalog des Verzehrbaren, gleichkommt. Im Kosmos des Provinzkrimis sind diätetische und multikulturelle Produkte weder Genuss-, noch Lebensmittel, sie gelten als nicht essbar.

Unter dem Aspekt der Interpretation von Nahrung als kulturelles Zeichen fungieren Essensordnungen zugleich als Ausdruck von und Mittel zur Aufrechterhaltung der jeweiligen sozialen Ordnung. Indem sie, besonders durch Essenstabus, zum inneren Zusammenhalt und zur Abgrenzung nach außen beitragen, sind sie ein wesentlicher Faktor der Konstitution von Gruppenidentität ${ }^{70}$. Die mit dem Begriff des „kulinarischen Handelns "71 bezeichnete performative Dimension von Ernährung wirkt, je nachdem, ob durch seinen Vollzug die der Gesellschaft eingeschriebenen Strukturen und Traditionen perpetuiert oder durchbrochen werden, stabilisierend oder subversiv ${ }^{72}$. Eine Essensordnung wie die in den analysierten Texten vorliegende, die konträr zu dominanten aktuellen Nutritionsprinzipien ausgerichtet ist, definiert dieserart die ihr zugeordnete Gesellschaft grundlegend als traditionalistisch und grenzt sie von modernistisch konnotierten Gesellschaftsformationen ab. Damit wird ein etwa seit dem ausgehenden 19. Jahrhundert evidentes Dispositiv angesprochen, das mit dem Prozess der Urbanisierung etablierte Gegensatzpaar Stadt - Land, welches klischeehafte Zuweisungen binärer Oppositionen mit sich führt, an prominenter Stelle die Korrelation von Metropole und Modernität versus Provinz

69. - Falk: SD, a.a.O., 21.

70. - Vgl. Eva Barlösius: Soziologie des Essens. Eine sozial- und kulturwissenschaftliche Einführung in die Ernährungsforschung, 2., völlig überarb. u. erw. Aufl., München, Weinheim, Juventa 2011 (Grundlagentexte Soziologie), 93-102.

71. - Klaus J. Mattheier: „Das Essen und die Sprache. Umrisse einer Linguistik des Essens", in: Wierlacher, Neumann, Teuteberg (Hg.): Kulturthema Essen, a.a.O., 245-255, hier 247.

72. - Vgl. ebd. 
und Nicht-Modernität ${ }^{73}$. Da, wie es der Eigenschaft von Stereotypen entspricht, dieses polare Deutungsschema trotz Veränderung und Ausdifferenzierung der Lebenswelt in Kraft geblieben ist ${ }^{74}$, bewirkt die Markierung eines fiktionalen Raums als anti- bzw. vormodern in diesem Kontext seine Wahrnehmung als Provinz. Die traditionalistische Konfiguration des Ernährungsdiskurses ist somit verantwortlich für die Definition der Raumkategorie, von Provinz schlechthin, die, ebenso wie umgekehrt, innerhalb der dichotomen Ordnung als Idealtypus dadurch bestimmt ist, dass sie nicht Stadt ist ${ }^{75}$.

Darauf aufbauend übernimmt der antimultikulturalistische Diskurs über seine antimodernistische Komponente hinaus die Stiftung der Identität der bereits als Provinz klassifizierten, innerhalb dieser Kategorie jedoch $\mathrm{zu}$ individualisierenden und von anderen Provinzräumen $\mathrm{zu}$ differenzierenden Region. Nahrungsmittel und Zubereitungsarten, die einen „regionaltypischen und eigenkulturellen Charakter"76 haben, „gehören als eigenkulturelle Werte zur ,kulturellen Identität' einer Person oder einer Gruppe" 77 und vermögen somit, erzählerisch ausgestaltet, eine solche fiktional erfahrbar $\mathrm{zu}$ machen. Zunächst errichtet die Konzentration auf ausschließlich für den süddeutschen ländlichen Raum spezifische Küche, die sprichwörtliche Hausmannskost, dessen Identität. Im Anschluss kommt die literarische Operationalisierung des soziologischen Phänomens der „Speisemeidung als Mittel soziokultureller Identifikation"78 zum Einsatz. Der Geschmack der Figuren in seiner Dimension als kulturstiftende Kategorie ${ }^{79}$ verweigert sich der beim Genuss nichtheimatlicher Speisen sinnlich erfahrenen Fremdheit ${ }^{80}$

73. - Vgl. Helmuth Kiesel: Geschichte der literarischen Moderne. Sprache, Ästhetik, Dichtung im zwanzigsten Jahrhundert, München, Beck 2004, 53-55; Norbert Mecklenburg: Erzählte Provinz. Regionalismus und Moderne im Roman, Königstein / Ts., Athenäum 1982, 16.

74. - Vgl. Dieter Burdorf, Stefan Matuschek: „Einleitung“, in: dies. (Hg.): Provinz und Metropole. Zum Verhältnis von Regionalismus und Urbanität in der Literatur, Heidelberg, Winter 2008, 9-13, hier 9.

75. - Vgl. Stefan Rehm: Stadt/Land. Eine Raumkonfiguration in Literatur und Film der Weimarer Republik, Würzburg, Ergon 2015 (Literatura. Wissenschaftliche Beiträge zur Moderne und ihrer Geschichte; Bd. 32), 40-41.

76. - Tolksdorf: „Das Eigene“, a.a.O., 190.

77. - Ebd., 188.

78. - Rudolf Weinhold: „Speisemeidung als Mittel soziokultureller Identifikation: das Exempel Pferdefleisch“, in: Hans Jürgen Teuteberg, Gerhard Neumann, Alois Wierlacher (Hg.): Essen und kulturelle Identität. Europäische Perspektiven (Kulturthema Essen; Bd. 2), 403-414, hier 403.

79. - Vgl. Gerhard Neumann: „Jede Nahrung ist ein Symbol. Umrisse einer Kulturwissenschaft des Essens", in Wierlacher, Neumann, Teuteberg (Hg.): Kulturthema Essen, a.a.O., 385-444, hier 388.

80. - Vgl. Alois Wierlacher: Vom Essen in der deutschen Literatur. Mahlzeiten in Erzähltexten von Goethe bis Grass, Berlin, Köln, Mainz u.a., Kohlhammer 1987, 100. 
und reagiert mit deren strikter Zurückweisung, was als Abgrenzung von jeglicher Alterität die spezifische regionale Identität affirmiert. Vermittels dieser Strategien wird der erzählte Raum mit massiv wirksamen kommunikativen Signalen ausgestattet, die ihn in einer über die bloße Festlegung des Handlungsortes weit hinausreichenden Intensität als Provinz wahrnehmbar machen und somit gattungsstiftende Funktion für den Provinzkrimi versehen. 